\title{
Caracterización de la implementación de herramientas de Lean Manufacturing: Estudio de caso en algunas empresas colombianas
}

\section{Characterization of the implementation of Lean Manufacturing tools: Case study in some Colombian companies}

\author{
Natalia Marulanda Grisales (Colombia) \\ nmarulandag@uniminuto.edu.co, \\ namagri@yahoo.es \\ Corporación Universitaria Minuto de Dios \\ Magíster en Ingeniería-Ingeniería Industrial
}

\author{
Gonzalo Emilio León (Colombia) \\ geleonr@escolme.edu.co, \\ gonzalo.leon@visionglobal.com.co \\ Institución Universitaria ESCOLME \\ Magíster en Negocios Internacionales
}

\section{Henry Helí González Gaitán (Colombia)}

henry.gonzalez@uniminuto.edu, henryheligg@gmail.com Corporación Universitaria Minuto de Dios

Magíster en Educación
Edgar Alejandro Hincapié Pizza (Colombia)

edgar.hincapie@uniminuto.edu, alejopizza@hotmail.com Corporación Universitaria Minuto de Dios Maestría en Administración (MBA)
25 DE NOVIEMBRE DE 2015

21 DE JUNIO DE 2016

5 DE JULIO DE 2016
PARa CitAR ESte artículo / to CITE thIS ARTICle

Marulanda Grisales, N., González Gaitán, H. H. León Rincón, G. E., \& Hincapié Pizza, E. A., (2016). Caracterización de la implementación de herramientas de Lean Manufacturing: Estudio de caso en algunas empresas colombianas, Poliantea, 12(22). 


\section{Resumen}

Aunque la aplicación de herramientas de lean manufacturing tiene un crecimiento sostenido a nivel mundial, en Colombia su uso aun es reducido. Por ende, el propósito del presente artículo es realizar un estudio de caso en cinco compañías ubicadas en Colombia que han implementado la filosofía lean a nivel productivo y administrativo. Para tal fin, se empleó una metodología de carácter cualitativo y cuantitativo, basada en entrevista e interlocución con los líderes de procesos.

Entre los principales hallazgos, se encontró que las herramientas más empleadas son las 5 s con el 100\% y TPM con el 80\%. La de menor uso es producción sincrónica con un $20 \%$. Aunque las cinco compañías han realizado capacitaciones en esta filosofía, solo el $40 \%$ del personal consultado conoce la definición de lean. No obstante, el $80 \%$ de las organizaciones participantes manifestaron tener programas de mejora que facilitan la motivación y empoderamiento del personal.

Palabras clave: cultura organizacional, estrategia corporativa, lean manufacturing, mejoramiento continuo.

\section{Summary}

Although the application of lean manufacturing tools has constantly grown globally, their use in Colombia is small. Therefore, the purpose of this paper is to conduct a case study in five companies, located in Colombia, that have implemented a lean philosophy at the productive and administrative level. To this end, a qualitative and quantitative methodology was used, based on interviews and dialogs with the people that lead the process.

Among the key findings, we found that the most commonly used tools were the 5 s with $100 \%$ and TPM with $80 \%$. The least used is synchronous production with $20 \%$. Although the five companies have provided training in philosophy, only $40 \%$ of the personnel interviewed knew the definition of lean. However, $80 \%$ of participating organizations have said they poses improvement programs that motivate and empower the personnel.

Clasificación JEL: L21, L6O, MI 1

Keywords: Organizational culture, corporate strategy, lean manufacturing, continuous improvement. 


\section{Introducción}

El desarrollo de metodologías de Además, requiere de un mecanismejora continua ha permitido que mo de coordinación entre todos los las organizaciones se vuelvan más miembros de la estructura organizacompetitivas en mercados con encional, de tal manera que se genere tornos altamente variables. Lo ancultura y compromiso en los proceterior ha propiciado el desarrollo sos de desarrollo e implementación. de estrategias y herramientas que Para tal fin, desde la estrategia corfacilitan la gestión de los procesos porativa se deben definir los meproductivos y administrativos, opcanismos de acción que faciliten la timizan recursos e incrementan los incorporación de la filosofía lean en niveles de eficacia, eficiencia y efeclas actividades diarias de los miemtividad. No obstante, las caractebros de la compañía. Lo anterior se rísticas propias de cada compañía, como el modelo de gestión, recursos y capacidades, pueden llegar a convertirse en barreras para el éxito de las herramientas que componen cada metodología.

logra con entrenamiento y capacitación, a fin de incrementar el conocimiento de los procesos y disminuir la resistencia al cambio por parte de los individuos.

Ahora bien, en Colombia exis-

Como alternativa aparece la filosofía lean manufacturing, la cual facilita la generación de valor a un determinado producto o servicio, a partir de la identificación y eliminación de mudas o desperdicios. ten algunos sectores que se destacan por su liderato en la implementación de lean manufacturing a nivel productivo y administrativo, como es el caso del sector automotriz y el metalmecánico. Sin embargo, la 
aplicación de estas metodologías aún es incipiente, se limita al uso de algunas herramientas. En ocasiones suele realizarse de manera empíri$\mathrm{ca}$, requiere del seguimiento constante de las curvas de aprendizaje y del compromiso de todos los miembros de la organización, incluyendo la alta dirección. Por este motivo, el objetivo de la presente investigación es realizar un estudio de caso en cinco compañías que se encuentran ubicadas en Colombia, con base en la caracterización de los elementos y particularidades que favorecieron el desarrollo de la filosofía lean manufacturing en ellas.

Así pues, el presente documento consta de cuatro secciones que se encuentran distribuidas de la siguiente manera. En primer lugar, se hará una ilustración conceptual de la metodología lean manufacturing y sus herramientas. En la siguiente sección, se abordará la metodología empleada en la investigación. A continuación, se exhibirán los resultados del estudio con respecto a las generalidades de aplicación de la filosofía lean, condiciones previas de las organizaciones, estructura cultural y motivacional, riesgos y barreras. Finalmente, se presentarán las respectivas conclusiones de la investigación.

\section{Lean manufacturing}

Los constantes cambios en las características de los mercados han generado un escenario en el cual las compañías buscan nuevas estrategias y metodologías, a fin de garantizar una ventaja competitiva. Dichos elementos deben propender por el mejoramiento de los procesos, productos y servicios, los cuales al ser replicados por los individuos se interiorizan como parte de la cultura organizacional. Así pues, se han desarrollado algunos modelos y filosofías en el área productiva, que pueden ser replicados a nivel administrativo con el fin de incrementar los niveles de eficiencia, eficacia y productividad global. Ahora bien, el lean manufacturing hace parte de estas metodologías y se basa en la mejora continua facilitando la optimización de recursos, satisfacción de los clientes internos y externos y mejora del desempeño de la compañía.

Lean significa emplear menos de todoslos recursosenla producción en masa (Wahab, Mukhtar y Sulaiman, 2013)"container-title":"Procedia Technology"',collection-title":"4th International Conference on Electrical Engineering and Informatics, ICEEI 2013'"'page":"'1292-1298','vo 
lume":"11"',source":"ScienceDirect" ,"abstract":"Lean manufacturing or also known as lean production has been one of the most popular paradigms in waste elimination in the manufacturing and service industry. Thus, many firms have grabbed the benefits to practice lean manufacturing in order to enhance quality and productivity. However, previous research shows that there are various sets of tools or techniques that had been adopted at a certain degree across firms according to their own understanding of lean manufacturing. The scenario resulted with varying leanness measures in order to measure lean practices. This paper describes a preliminary study in developing a conceptual model to measure leanness in manufacturing industry. Thorough literature survey, books and report analysis contribute to the main preliminary analysis of this study. The most common tools or techniques and their usefulness have been investigated. In this research, a conceptual model for leanness measurement in the manufacturing industry has been developed and designed in two main levels, namely the dimensions and the factors. There are seven main dimensions in measuring leanness in lean manufacturing practices such as manufacturing process and equipment, manufacturing planning and scheduling, visual information system, Supplier relationship, customer relationship, workforce and product development \&amp; technology. In addition, the model also shows how lean dimensions in the manufacturing system relate to eight types of wastes."'DOI":"10.1016/j. protcy.2013.12.327"',ISSN":"22120173',"journalAbbreviation":"Proc edia Technology'”,'author":[[“'famil y":"Wahab"',given":"Amelia Natasya Abdul"\},\{“family":"Mukhtar",'giv en":"Muriati”\},\{“family":"Sulaiman" ,"given":"Riza"\}],"issued":\{“date-par ts":[[“2013"]]\}\}\}],"schema":"https:// github.com/citation-style-language/schema/raw/master/csl-citation. json"\} . Asimismo, busca eliminar la ineficiencia de las operaciones diarias que no están generando valor a los productos y servicios (Conner, 2006). De esta manera, se obtienen mejoras continuas en los sistemas productivos al disminuir los costos derivados de la eliminación de desperdicios (Santos, Vieira y Balbinotti, 2015)"container-title":"Procedia Manufacturing","collectiontitle":"6th International Conference on Applied Human Factors and 
Ergonomics (AHFE 2015. No obstante, para que la implementación de la filosofía lean sea exitosa, la alta dirección debe participar de manera activa en el programa, proporcionar entrenamiento y capacitación, tener un plan en el largo plazo y evaluarlo día a día, asignar recursos y compartir las ganancias, aplicar técnicas y herramientas lean (Netland, 2015).

Bajo este aspecto, el Lean Manufacturing como componente de los sistemas de mejoramiento continuo, permite a las organizaciones desarrollar una excelencia operacional a partir del uso adecuado de los recursos empresariales, y la disminución de costos y tiempos de producción (Arrieta, Botero y Romano, 2010), (Alukal y Manos, 2006). Además, el lean busca satisfacer las necesidades de los clientes cuando lo quieren, donde lo deseen, a un precio competitivo, en las cantidades y variedades que ellos quieran, y siempre de excelente calidad (Alukal y Manos, 2006; Villaseñor y Galindo, 2007). El nacimiento de la filosofía lean manufacturing se dio a partir del Sistema de Producción Toyota - TPS por sus siglas en inglés(Pérez Rave, 2011), (Madariaga, 2013), como resultado del trabajo y las iniciativas de Eiji Toyoda, quien quería mejorar el proceso manufacturero de Toyota, hasta igualarlo con la productividad de la compañía Ford (Villaseñor y Galindo, 2007).

Análogamente, el lean manufacturing ayuda a los líderes de proceso a identificar y eliminar los desperdicios o mudas, los cuales corresponden a aquellas actividades que no aportan valor al producto (Madariaga, 2013; Rajadell \& Sánchez, 2010). Son varios los autores que coinciden con la existencia de siete tipos de desperdicios o mudas (Alukal y Manos, 2006; Pérez, 2011; Villaseñor y Galindo, 2007), que interfieren directamente en el desempeño operacional de las organizaciones. Estas mudas son: la sobreproducción, espera, transportes innecesarios, movimientos innecesarios, sobreprocesamiento, inventarios y defectos. De aquí que el mayor objetivo de la filosofía lean manufacturing, en cualquier tipo de compañía, sea eliminar todos los anteriores desperdicios (Alukal y Manos, 2006).

No obstante, el desarrollo de herramientas lean trae una serie de beneficios que van más allá de la eliminación de mudas. Entre estos se 
encuentran: mejoras a nivel operacional, administrativo y estratégico (Kilpatrick, 2003). Además, facilitan la incursión de las compañías en un mercado global, al adaptarse a los cambios tecnológicos, implementación de estándares de calidad y cumplir con las expectativas de los clientes (Alukal y Manos, 2006). Dichas herramientas se abordarán a continuación.

\section{Herramientas Lean}

Algunas de las herramientas y técnicas usadas para introducir, mantener y mejorar el sistema lean se encuentran en un diagrama conocido como "Casa del Sistema de Producción Toyota” (Hernández y Vizán, 2013), las cuales se encargan de sustentar los procesos que permiten alcanzar las metas de mejora (Ver Tabla 1).

Tabla 1. Herramientas Lean Manufacturing

\begin{tabular}{|c|c|c|}
\hline Herramienta & Descripción & Fuente \\
\hline Just in Time(JIT) & $\begin{array}{l}\text { - Entregar al cliente lo que desea, en la cantidad que } \\
\text { desea y exactamente como lo desea. } \\
\text { - Reduce buena parte del desperdicio, a partir del flujo, } \\
\text { calidad e intervención de los empleados. } \\
\text { - Reduce plazos de entrega, niveles de inventario, } \\
\text { mejora la calidad. } \\
\text { - Proporciona operaciones fluidas y retroalimentación } \\
\text { inmediata. }\end{array}$ & $\begin{array}{l}\text { (Cuatrecasas, 2006); (Hay, 1989); (Gaither, } \\
\text { 2000); (Heizer y Render, 2007) }\end{array}$ \\
\hline Células de manufactura & $\begin{array}{l}\text { - Reconfiguración de operaciones para mantener un } \\
\text { flujo continuo. } \\
\text { - Permite la clasificación por familias, ya que los } \\
\text { productos tienen características similares. } \\
\text { - Mejora la comunicación y utilización de personas y } \\
\text { equipos. } \\
\text { - Considera la formación, disposición y secuencia de } \\
\text { las máquinas. }\end{array}$ & $\begin{array}{l}\text { (Villaseñor y Galindo, 2007); (Salazar, } \\
\text { Vargas, Añasco y Orejuela, 2010); (Cardona, } \\
2013 \text { ); (Chang, Wu y Wu, 2013)only few } \\
\text { articles have focused on more practical and } \\
\text { complicated problems that simultaneously } \\
\text { consider the three critical issues in the } \\
\text { cMS-design process, i.e., cell formation, cell } \\
\text { layout, and intracellular machine sequence. } \\
\text { In this study, a two-stage mathematical } \\
\text { programming model is formulated to integrate } \\
\text { the three critical issues with the consideration } \\
\text { of alternative process routings, operation } \\
\text { sequences, and production volume. Next, } \\
\text { because of the combinatorial nature of } \\
\text { the above model, an efficient tabu search } \\
\text { algorithm based on a generalized similarity } \\
\text { coefficient is proposed. Computational results } \\
\text { from test problems show that our proposed } \\
\text { model and solution approach are both } \\
\text { effective and efficient. When compared to the } \\
\text { mathematical programming approach, which } \\
\text { takes more than } 112 \mathrm{~h} \text { (LING0; (Jajodia, } \\
\text { Minis, Harhalakis y Proth, 1992) which has } \\
\text { been successfully applied for the solution of } \\
\text { combinatorial problems. }\end{array}$ \\
\hline
\end{tabular}




\section{- Caracterización de... - Natalia M.Grisales, Henry H. González, Gonzalo E. León y Edgar A. Hincapié}

\begin{tabular}{|c|c|c|}
\hline Herramienta & Descripción & Fuente \\
\hline & & $\begin{array}{l}\text { A major advantage of this technique is the } \\
\text { insensitivity of the final solution to the initial } \\
\text { conditions. In addition, some important } \\
\text { practical issues such as intra-cell layout of } \\
\text { machines in predetermined configurations } \\
\text { (e.g., row-wise or circular arrangements }\end{array}$ \\
\hline Jidoka & - Automatización teniendo en cuenta al ser humano. & $\begin{array}{l}\text { (Hernández y Vizán, 2013); (Villaseñor y } \\
\text { Galindo, 2007) }\end{array}$ \\
\hline Poka Yoke & $\begin{array}{l}\text { - A prueba de errores, respetando la inteligencia de los } \\
\text { trabajadores. } \\
\text { - Prevenir la producción de defectos a través de la } \\
\text { detección temprana de errores. }\end{array}$ & $\begin{array}{l}\text { (Villaseñor y Galindo, 2007); (Hernández y } \\
\text { Vizán, 2013) }\end{array}$ \\
\hline Kaizen o mejora continua & $\begin{array}{l}\text { - Cultura de mejora continua sostenible. } \\
\text { - Involucra a toda la estructura organizacional y fiene } \\
\text { costos relativamente bajos. } \\
\text { - Forma líderes para proponer mejoras en el largo plazo. }\end{array}$ & (Alukal y Manos, 2006); (Imai, 2012) \\
\hline $\begin{array}{l}\text { Value Stream Mapping } \\
\text { (VSM) }\end{array}$ & $\begin{array}{l}\text { - Mapa en el que se especifica la cadena de valor de } \\
\text { la organización tanto en áreas productivas como } \\
\text { gerenciales. } \\
\text { - Identifica el flujo de procesos y los desperdicios. } \\
\text { - Da respuesta a problemáticas de comunicación, } \\
\text { personal, material, equipos y procesos. }\end{array}$ & $\begin{array}{l}\text { (Rother y Shook, 2003); (Beau Drew, } \\
\text { 2004); (Sullivan, McDonald y Van Aken, } \\
\text { 2002)the large fixed costs of production are } \\
\text { depreciation-intensive because of huge capital } \\
\text { investments made in high-volume operations. } \\
\text { These fixed costs are spread over large } \\
\text { production batch sizes in an effort to minimize } \\
\text { the total unit costs of owning and operating } \\
\text { the manufacturing system. As an alternative } \\
\text { to \"batch-and-queue,)" high-volume, and } \\
\text { inflexible operations, the principles of the } \\
\text { Toyota Production System (TPS; (Nash y } \\
\text { Poling, 2008) }\end{array}$ \\
\hline $5 s$ & $\begin{array}{l}\text { - Conformado por: Seiri (Clasificación), Seiton (Orden), } \\
\text { Seiso (Limpieza), Seiketsu (Estandarización); } \\
\text { Shitsuke (Disciplina). } \\
\text { - Evita problemas derivados del desorden y la falta de } \\
\text { instrucciones. } \\
\text { - Proporciona bienestar, disciplina y un ambiente } \\
\text { armónico. }\end{array}$ & $\begin{array}{l}\text { (Villaseñor y Galindo, 2007); (Rajadell y } \\
\text { Sánchez, 2010); (Pérez Rave, 2011); } \\
\text { (Villaseñor y Galindo, 2011) }\end{array}$ \\
\hline $\begin{array}{l}\text { Single-Minute Exchange of } \\
\text { Die (SMED) }\end{array}$ & $\begin{array}{l}\text { - Preparación de la producción y cambios útiles en un } \\
\text { solo dígito. } \\
\text { - Elimina ajustes y estandarización mediante la } \\
\text { instalación de nuevos mecanismos, plantillas y } \\
\text { anclajes funcionales. }\end{array}$ & $\begin{array}{l}\text { (Villaseñor y Galindo, 2007); (Hernández y } \\
\text { Vizán, 2013) }\end{array}$ \\
\hline \multirow[t]{2}{*}{$\begin{array}{l}\text { Total Productive } \\
\text { Maintenance (TPM) }\end{array}$} & $\begin{array}{l}\text { - Busca la mejora continua, eficiencia del proceso } \\
\text { e involucra a todo el personal y departamentos } \\
\text { organizacionales. }\end{array}$ & $\begin{array}{l}\text { (Sánchez, 2007); (Cuatrecasas, 2010); } \\
\text { (Hernández y Vizán, 2013) }\end{array}$ \\
\hline & $\begin{array}{l}\text { - Previene pérdidas en todas las operaciones de la } \\
\text { empresa. } \\
\text { - Maximiza la efectividad y alarga la vida del equipo. }\end{array}$ & \\
\hline Kanban & $\begin{array}{l}\text { - Etiqueta que comunica información sobre el flujo del } \\
\text { producto, para nivelar la producción. }\end{array}$ & (Monden, 1996) \\
\hline
\end{tabular}




\begin{tabular}{|c|c|c|}
\hline Herramienta & Descripción & Fuente \\
\hline Gestión visual & $\begin{array}{l}\text { - Tener a la mano toda la información del sistema para } \\
\text { facilitar su comprensión. } \\
\text { - Empodera y genera sentido de pertenencia en los } \\
\text { empleados. } \\
\text { - Demarca áreas, materiales, productos, equipos, } \\
\text { programas de producción. } \\
\text { - Emplea indicadores. }\end{array}$ & $\begin{array}{l}\text { (Alukal y Manos, 2006); (Hernández y } \\
\text { Vizán, 2013) }\end{array}$ \\
\hline Six Sigma & $\begin{array}{l}\text { - Mejora la efectividad y eficiencia de los procesos al } \\
\text { controlar las variaciones. } \\
\text { - Implementan iniciativas de mejora para garantizar } \\
\text { precisión y cumplimiento de las expectativas del } \\
\text { cliente. } \\
\text { - El número de defectos máximos permisibles es de } \\
3,4 \text { en un millón. }\end{array}$ & $\begin{array}{l}\text { (Eckes, 2006); (Zavalla, 2010); (Youssouf, } \\
\text { Rachid e lon, 2014)housing, automobile, } \\
\text { manufacturing industry and construction } \\
\text { (factories, housing, highway, subway, tram, } \\
\text { etc.; (Aguirre, 2010)Medir, Analizar, Mejora } \\
\text { y Controla }\end{array}$ \\
\hline
\end{tabular}

Fuente. Elaboración propia

La implementación de las anteriores herramientas ha de realizarse de manera secuencial o paralela en aquellas áreas organizacionales que así lo requieran. El uso inadecuado de recursos en cuanto a tiempo y cantidad, puede llevar al fracaso durante su ejecución. Sin embargo, resulta beneficioso realizar benchmarking entre compañías cuyas razones sociales o de sectores sean similares, con el fin de identificar aquellas características de las herramientas lean que pudiesen tener un impacto beneficioso en una determinada organización en concordancia con el direccionamiento estratégico de las mismas.

Una de las herramientas de diagnóstico que proporciona el Lean Manufacturing y que se encuentra en la Tabla 1 es VSM o "gestión Visual".
Esta herramienta permite, desde su aplicación, visualizar a dónde quiere llegar la organización y/o cada uno de los procesos a ser intervenidos, qué elementos y recursos son necesarios y a quién se debe empoderar para el logro de los objetivos.

Elementos que serán empleados en la caracterización de la implementación de herramientas de lean manufacturing

La adopción de una nueva metodología o estrategia en una organización se debe a las decisiones de la alta dirección, a las tendencias observadas en el mercado y a los avances en procesos óptimos de producción. Sin embargo, no se puede garantizar el éxito de su aplicación, si las compañías no cuentan con una serie de recursos y capacidades que les sirvan como estructura 
de soporte y apoyo. Esto es lo que se define en la presente investigación como generalidades.

En primer lugar, se debe identificar si la decisión de implementación se deriva de la estrategia corporativa o una unidad de negocio en particular. Por su parte, la estrategia corporativa les permite a las organizaciones alinear sus recursos más valiosos para satisfacer las necesidades individuales de los clientes, a fin de incrementar su lealtad para la adquisición de productos $\mathrm{y}$ servicios (Garri, Konstantopoulos y Bekiaris, 2013). Además, la estrategia corporativa evalúa aquellas posibles alternativas de negocio en las cuales la compañía puede invertir, teniendo en cuenta información del pasado y la incertidumbre del mercado (Maroto, 2007).

Con respecto a la estrategia de negocios, busca alcanzar un posicionamiento competitivo y sostenible de las empresas por medio del cumplimiento de los lineamientos establecidos por la corporación, teniendo en cuenta las capacidades y recursos que se han destinado a cada unidad de negocios (Hax, 2004). Siendo su principal objetivo, la obtención de una ventaja competitiva (David, 2003).
Ahora bien, si se detectan fallas en las decisiones estratégicas de las organizaciones, se deben implementar metodologías y alternativas de mejora continua, con el objetivo de optimizar los procesos y recudir las restricciones del mercado. Así pues, el mejoramiento continuo o kaizen no se refiere exclusivamente a la calidad, sino también a los procesos. Además, el mejoramiento continuo requiere del mantenimiento de buenos canales de comunicación entre directivos y empleados, y del empoderamiento del personal a cargo de un determinado proceso, pues son ellos los que están en capacidad de identificar los puntos críticos que pueden mejorarse (Krajewski y Ritzman, 2000); (Maarof y Mahmud, 2016).

Teniendo en cuenta el papel que adquiere el personal en el desarrollo de metodologías de mejora continua, es importante delimitar y redefinir las posiciones de poder en los procesos organizacionales. En tal caso, el empoderamiento en los lugares de trabajo se encuentra influenciado por las metas, competencias, autodeterminación y beneficios a obtener por cada trabajador (Sun, Zhang, Qi y Chen, 2012). El empoderamiento tiene un efecto positivo 
en la productividad, calidad, innovación, satisfacción laboral y motivación del empleado (Tessem, 2014).

Los anteriores conceptos pueden agruparse en un término que proporciona una visión holística sobre la gestión y mejora empresarial. Dicho término se refiere a la cultura organizacional, la cual involucra una serie de comportamientos y valores, que son distribuidos y adoptados por los individuos de una compañía (Paredes, 2005). Como se verá en el desarrollo de las siguientes secciones, la cultura organizacional adquiere un papel de desempeño transversal en cada uno de los aspectos que se evaluaron en el estudio de caso. Lo anterior va en concordancia con la adopción de las herramientas lean manufacturing, pues el éxito de esta filosofía depende en gran medida de la actitud y compromiso de los individuos.

\section{Metodología}

La metodología empleada posee un alcance descriptivo y un enfoque cualitativo, en el cual se pretende realizar una caracterización de los componentes empresariales que favorecieron la implementación de algunas herramientas de la filosofía lean manfufacturing. Se basa en cinco estudios de caso de organizaciones colombianas ubicadas en el Valle de Aburrá, las cuales han empleado dichas herramientas. Así pues, se llevó a cabo el levantamiento de información primaria mediante la ejecución de un muestreo no probabilístico por conveniencia. Para la recolección de los datos, se diseñó un formulario con preguntas abiertas, lo cual facilitó la realización de entrevistas a profundidad e interlocución con los líderes de proceso de dichas compañías, los cuales fueron seleccionados por su intervención directa en la toma de decisiones al momento de implementar herramientas lean, además de ser los encargados de realizar vigilancia y control a dicho proceso.

Las organizaciones y los dos miembros del personal participante en la investigación fueron: Siemens S.A (Gerente General de la Fábrica de Transformadores Siemens en Colombia, Directora de Excelencia Operacional y Estrategias de Mercadeo); Incolmotos Yamaha S.A. (Coordinador de TPM (Total Productive Maintenance, Jefe de Mejoramiento Continuo); Único Interior S.A.S. (Director de Operaciones, Jefe de Producción). Igualmente, se 
consultaron dos compañías que por solicitud expresa no quieren que su nombre sea publicado: Compañía dedicada a la fabricación de GasoElectrodomésticos (Jefe de Manufactura) y Compañía del sector textil (Asistente de Aseguramiento de Procesos y Jefe de Gestión Humana).

El formulario está conformado por cinco secciones. En primer lugar, consta de un conjunto de preguntas generales sobre las metodologías de gestión de la empresa, definición de lean manufacturing, herramientas, fases de ejecución, beneficios y dificultades. En la segunda y tercera etapa, se indagó por los componentes motivacionales, organizacionales y de seguimiento que permitieron la implementación del lean manufacturing. Con la cuarta sección se pretendía indagar por las características y requerimientos previos a la adopción del lean. Finalmente, el formulario buscaba conocer el conjunto de riesgos y barreras a las que se vieron expuestas las compañías una vez decidieron aplicar herramientas lean.

Por otro lado, la información secundaria se obtuvo a partir de la revisión detallada de bases de datos, repositorios institucionales, archivos, informes y revistas especializadas en el área. De esta manera, con la tabulación de todos los datos e información recabada, se procedió con la realización de una caracterización de las cinco compañías consultadas con el objetivo de identificar las particularidades conjuntas de la aplicación de la filosofía lean. Los resultados derivados se presentarán a continuación.

\section{Resultados}

La caracterización de las cinco compañías que participaron en el estudio de caso se encuentra dividida en cuatro áreas funcionales, las cuales se derivan de la segmentación establecida en el formulario empleado en las consultas y entrevistas que fueron realizadas a los líderes de procesos. Dichas áreas son: generalidades de las organizaciones, condiciones previas, estructura cultural y motivacional, riesgos y barreras.

\section{Generalidades de las organizaciones}

De las cinco compañías que participaron en el estudio de caso, el $60 \%$ operan en Colombia como filiales de empresas extranjeras. Por ende, su estrategia corporativa se desprende de las acciones y directrices que son proporcionadas por las diferentes Casas Matrices. Sin 
embargo, los líderes de procesos aclararon que debido a la composición de los recursos organizacionales y a las características culturales, las estrategias de negocio y funcional tienden a desvincularse de los parámetros previamente establecidos. Lo anterior ha permitido que dichas compañías logren competir de manera ventajosa y sostenida en los mercados en los cuales se desenvuelven: textil, automotriz, transformadores y electrodomésticos.

De manera particular, el $40 \%$ de las compañías participantes consideran como su principal impulsor y eje central de su misión el desarrollo del capital humano. Otro de sus propósitos son: mantenimiento de la calidad, mejoramiento continuo, disminución de tiempos muertos, incremento de la eficiencia, eficacia y rentabilidad. Los líderes de procesos aclaran, además, que debe existir un compromiso global de la alta gerencia para soportar las diferentes decisiones que involucran a todo el sistema. Sin lugar a dudas, todos los cambios propuestos redundan en la disminución de costos y recursos que permiten maximizar el margen de ganancia.

Si bien el propósito del presente estudio consistió en realizar una caracterización de la aplicación de herramientas lean manufacturing, cuando se les preguntó a los líderes de proceso por la definición de dicho término, solo dos de ellos proporcionaron una explicación del concepto. Así pues, este se definió como orden y compromiso de todos los miembros de la organización, incluyendo la alta dirección. También describieron la filosofía lean como una práctica que involucra un alto contenido de sentido común, sistemática y metodología, la cual requiere del involucramiento de los dueños de los procesos. Lean es complacer la demanda del cliente, diseñar un proceso productivo que sea capaz de responder a esa demanda, en términos de personas y materiales.

Con respecto a las herramientas lean que han sido empleadas por las compañías del estudio, las de mayor uso son las $5 \mathrm{~s}$ con el $100 \% \mathrm{y}$ TPM con el 80\% (Ver Tabla 2.). La de menor uso es producción sincrónica con un $20 \%$, ya que es la que requiere de un mayor número de recursos y compromiso de todos los individuos, pues dicha herramienta pretende lograr una coordinación de todos los ciclos de producción e incrementar el desempeño global de todo el sistema. 
Tabla 2. Uso de herramientas de lean manufacturing en las compañías objeto de estudio

\begin{tabular}{|l|c|c|c|c|c|c|c|c|c|c|c|}
\hline \multicolumn{1}{|c|}{ EMPRESA } & $5 S$ & Kanban & Kaizen & Six Sigma & JIT & SMED & $\begin{array}{c}\text { Celdas de } \\
\text { Manufactura }\end{array}$ & $\begin{array}{c}\text { Gestión } \\
\text { Visual }\end{array}$ & VSM & TPM & $\begin{array}{c}\text { Producción } \\
\text { Sincrónica }\end{array}$ \\
\hline SIEMENS S.A. & $X$ & & $X$ & $X$ & $X$ & $X$ & $X$ & $X$ & & $X$ & \\
\hline ÚNICO INTERIOR S.A.S. & $X$ & & & & $X$ & $X$ & $X$ & $X$ & $X$ & $X$ & \\
\hline ABCD & $X$ & & & & & & & & & & \\
\hline INDUSTRIAS HABC & $X$ & $X$ & & $X$ & & & & $X$ & $X$ & $X$ & \\
\hline INCOLMOTOS YAMAHA S.A. & $X$ & $X$ & $X$ & $X$ & & $X$ & $X$ & $X$ & $X$ & $X$ & $X$ \\
\hline Porcentaje aplicación & 1 & 0,4 & 0,4 & 0,6 & 0,4 & 0,6 & 0,6 & 0,8 & 0,6 & 0,8 & 0,2 \\
\hline
\end{tabular}

Fuente. Elaboración propia

En un comienzo, la implementación de las anteriores herramientas se realizó de manera paulatina en diferentes áreas de las compañías, donde los componentes de la filosofía lean fuesen necesarios. Es decir, que no todas las herramientas se implementaron de manera lineal en todas las áreas organizacionales. Los líderes de las compañías indagadas empezaron por el área productiva, pues los procesos que allí se desarrollan presentan menores inconvenientes al momento de ser estandarizados. Posteriormente, el $80 \%$ de las organizaciones del estudio trasladaron la filosofía lean a los niveles administrativos.

No se encontraron diferencias relevantes en la formación de los empleados de las organizaciones del estudio de caso, ya que el nivel mínimo de educación es bachillerato, seguido por la formación técnica y tecnológica, finalizando con el nivel profesional y de posgrado. Se aclara que para la implementación de la filosofía lean, en las cinco organizaciones estudiadas, todos los miembros que intervinieron en el proceso recibieron capacitaciones en las herramientas sin importar su nivel de formación.

Es importante aclarar que no es fácil identificar el nivel de madurez de las herramientas lean manufacturing en las organizaciones del estudio, ya que las motivaciones e impactos varían de una a otra. Lo anterior se determina por el contexto en el cual se desenvuelve cada organización. Por esto, en el siguiente apartado se abordarán las condiciones y características previas de las compañías estudiadas que favorecieron el desarrollo de la filosofía lean. 


\section{Condiciones previas}

La decisión de adopción e implementación de la filosofía lean manufacturing al interior de una compañía conlleva un conjunto de requisitos y condiciones previas que determinarán el éxito de dicha filosofía en términos de tiempo, recursos y resultados operacionales. A su vez, dichos resultados surgen del compromiso adquirido por cada uno de los individuos de la organización, incluyendo a los miembros de la alta dirección.

Dentro de esas condiciones previas existen un conjunto de razones iníciales que favorecieron la implementación de la filosofía de lean manufacturing, en las organizaciones que participaron en el estudio de caso (Ver Figura 1)

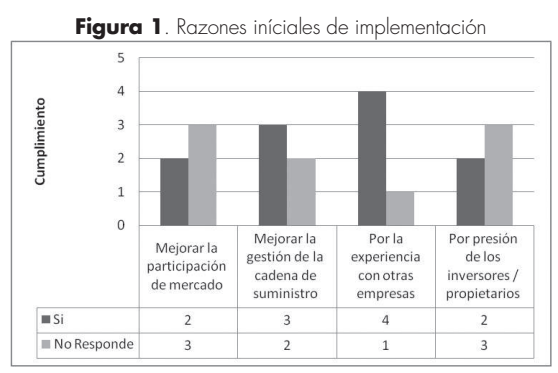

Fuente. Elaboración propia

Se advierte que el $80 \%$ de las compañías decidieron implementar manufactura esbelta, realizando benchmarking y teniendo en cuenta metodologías, herramientas y resultados que obtuvieron otras compañías como Interconexión Eléctrica S.A. (ISA) y Sociedad de Fabricación de Automotores S.A. (SOFASA). Además, tuvieron en cuenta la formación recibida por los líderes de proceso y su experiencia profesional previa en otras instituciones.

Hasta cierto punto, las directrices proporcionadas por algunas casas matrices obligaron que dos filiales en Colombia incorporaran las herramientas de manufactura esbelta en sus procesos productivos y en su estrategia de mejoramiento continuo. De manera particular, los líderes consultados manifestaron que su interés inicial no era mejorar la cuota de participación en el mercado a partir del desarrollo de la filosofía lean. Por su parte, el 60\% consideran la gestión de la cadena de suministro, y en especial el desarrollo de los proveedores, como uno de los aspectos claves para garantizar el éxito de las herramientas de manufactura esbelta.

\section{Estructura cultural y motivacional} La cultura organizacional se refiere al conjunto de creencias, normas 
$\mathrm{y}$ valores que tienen los individuos de una organización y que permiten que esta se distinga de las demás compañías (Fernández-Ríos y Sánchez, 1997). Si bien los elementos de la cultura organizacional suelen ser intangibles, los resultados de su adecuada gestión se reflejan en el posicionamiento que alcanzan las compañías. El factor humano como principal elemento de la cultura merece un tratamiento especial al momento de incorporar una nueva herramienta en los procedimientos empresariales.

Inicialmente, en las empresas consultadas, solo los líderes de proceso y algunos miembros de la dirección participaron en el proceso de implementación. Lo anterior se da porque el personal seleccionado para este rol debe poseer un gran conocimiento sobre los procesos y áreas que van a ser intervenidas. Así pues, los cargos que participaron en la etapa inicial en las compañías objeto de estudio son: jefes de producción y calidad, coordinador de TPM, jefes de área e ingenieros especializados. Igualmente, dentro de dichos cargos también se incluyó un conjunto de operarios expertos.

Con respecto al entrenamiento que se impartió para la incorporación de la filosofía lean en las compañías objeto de estudio, el 100\% de los líderes de proceso manifestaron que el personal involucrado en el proceso recibió capacitación en las herramientas de manufactura esbelta. No obstante, la formación que se impartió en una de las empresas se realizó de manera empírica e informal, sin contar con el apoyo de entidades certificadas en la enseñanza de herramientas lean. A su vez, el $40 \%$ de estas organizaciones recibió apoyo por parte de las casas matrices en el proceso de capacitación y formación, donde el learning by doing es un componente fundamental del proceso de aprendizaje.

Ahora bien, el sostenimiento de la filosofía lean requiere de capacitaciones constantes a los individuos que participan en los diferentes procesos productivos y administrativos. De aquí que las cinco compañías consultadas se encarguen de realizar evaluaciones periódicas a todos los miembros de la organización, tanto a los individuos que llevan cierto tiempo desempeñándose en funciones lean, como aquel personal nuevo que ingresa a la compañía. El objetivo es garantizar el sostenimiento de las curvas de aprendizaje. 
Las cinco compañías objeto de estudio se encargan de medir los impactos de las capacitaciones en herramientas lean manufacturing en la evaluación de desempeño del personal, cuyos resultados se están revisando de manera constante a fin de incorporar la mejora continua como parte de la cultura empresarial. Asimismo, una de las organizaciones realiza seguimiento permanente a las curvas de aprendizaje de cada uno de sus funcionarios, para lo cual se encargó de contratar una compañía outsourcing a fin de no descuidar las funciones de su objeto social.

Por otro lado, dos de las compañías consultadas aclaran que los individuos de una organización ven la implementación de la filosofía lean como sus líderes y directivos la vean. Lo anterior implica el desarrollo de mecanismos de coordinación que permitan el trabajo y acompañamiento constante de la alta dirección y de las casas matrices en cada una de sus filiales.

Con todo esto, el componte motivacional de los individuos puede llegar a tener repercusiones en los objetivos organizacionales, ya sea en términos de eficiencia, eficacia o productividad. Dicha motivación puede definirse como una conducta que nace de una determinada necesidad, la cual se dirige y orienta hacia el logro de una meta (Guillén, 2013). Para el caso específico del estudio, la incorporación de las herramientas lean en las organizaciones requirió de un esfuerzo adicional de los líderes de proceso, a fin de rescatar las mejores características individuales y conjuntas de los empleados.

En el estudio de caso se analizaron tres componentes motivacionales: salud ocupacional, liderazgo $\mathrm{y}$ trabajo en equipo. Con respecto a la salud ocupacional, el $40 \%$ de las compañías consideran las condiciones ergonómicas de los puestos de trabajo necesarias para garantizar la comodidad y mejorar el desempeño de cada empleado. En términos de liderazgo, más allá del papel que desempeñan los líderes de procesos y los directivos, en las organizaciones consultadas surgieron líderes naturales quienes ayudaron a facilitar la apropiación de las herramientas de manufactura esbelta. A su vez, el trabajo en equipo permitió compensar las falencias de los individuos en cada proceso.

De manera específica, el $80 \%$ de las compañías participantes en 
el estudio de caso manifestaron tener programas de mejora que buscan potenciar el involucramiento del personal en la optimización de procesos. Entre estos programas se encuentran: 3I (ideas, iniciativas e innovación), 3 Botones (A, B y C), y programas generales de mejora. Los anteriores programas brindan incentivos monetarios y en especie a aquellos individuos o conjunto de empleados que presentaron soluciones alternativas a problemas, las cuales fueron exitosas. Sin embargo, una de las empresas consultadas no brinda incentivos porque quiere que el programa se desarrolle de manera natural entre los empleados.

Es de aclarar que la incorporación de esta filosofía (lean manufacturing) en la cultura organizacional, presenta una serie de riesgos y barreras que pueden retrasar la consecución de los resultados operacionales. Dichos elementos se abordarán en la siguiente sección.

\section{Riesgos y barreras}

Desde las estrategias corporativas y de negocios se deben tener en cuenta los posibles obstáculos y contratiempos que pudiesen llegar a presentarse en la incorporación de una nueva metodología o herramienta en el quehacer organizacional. Lo anterior se asocia a aquellas características económicas, físicas y humanas que afectan el desarrollo y sostenibilidad de las nuevas estrategias que se integran a las compañías. Cuando se les preguntó a los líderes de procesos por los costos en los que se debió incurrir para el desarrollo de la manufactura esbelta, ninguna de las compañías consultadas contó con una cifra exacta de la inversión. El 40\% de ellas suministraron los montos de los procesos de capacitación y formación, obviando aquellos valores relacionados con infraestructura, materiales, maquinaria y equipo. No obstante, los líderes aclararon que se podía incurrir en dichos costos, si se demuestra que estos pueden ser suplidos por las ganancias operacionales derivadas de implementación de la filosofía lean, o por el ahorro de recursos.

Ahora bien, el reto más importante del lean manufacturing es lograr ser flexibles frente a las condiciones cambiantes que son impuestas por el mercado y por los clientes internos. El 60\% de las empresas del estudio de caso manifestaron situaciones en las cuáles el personal empezó a afectar la sostenibilidad del 
sistema, porque se relajaban y no tenían sentido de pertenencia con la aplicación de las nuevas herramientas. Para solucionar este inconveniente, las compañías empezaron a realizar evaluaciones a los empleados y seguimiento individual a las curvas de aprendizaje.

Igualmente, se presentó resistencia al cambio en las cinco organizaciones consultadas, porque los empleados no entendían que la filosofía lean debía empezar a hacer parte de su cultura y actividades diarias. Este inconveniente se solucionó con un acompañamiento constante por parte de los líderes de proceso y los miembros de la alta dirección. Asimismo, se incorporaron sistemas de capacitación e incentivos, los cuales lograron motivar al personal y convertir la filosofía de lean manufacturing en un componente de la cultura empresarial.

\section{Conclusiones}

En las cinco organizaciones que participaron en el estudio de caso se evidenció un desconocimiento general de la definición del término de lean manufacturing, pues únicamente el $40 \%$ de los líderes de proceso entrevistados lograron describir dicho término. Si bien el desconocimiento de la definición conceptual no fue impedimento para que las organizaciones decidieran adoptar la filosofía lean, la falta de comprensión puede producir retardos en la optimización y coordinación de los diferentes recursos organizacionales, afectando la incorporación de la filosofía manufactura esbelta a la cultura organizacional y disminuyendo los niveles de eficiencia, eficacia y productividad.

El $60 \%$ de las organizaciones entrevistadas concluyó que los materiales y sub ensambles proporcionados por sus proveedores afectan directamente su cadena productiva. Esta razón motivó a las mismas a extender la aplicación de la filosofía lean a los procesos productivos de sus proveedores, suministrando capacitación, realizando auditorías y seguimientos, lo que impacta directamente en el logro de los objetivos propios.

Aunque la decisión de implementación de la filosofía lean se dio por direccionamiento de las casas matrices en un $40 \%$, y por realización de benchmarking en un $60 \%$, es fundamental que el personal que intervenga en dichos procesos posea un conocimiento exhaustivo de los 
procedimientos que van a ser intervenidos. Asimismo, para asegurar el sostenimiento de la manufactura esbelta se debe realizar capacitación, seguimiento y acompañamiento constante a todos los miembros de la organización. Es decir, se debe vigilar de forma concienzuda tanto la curva de aprendizaje individual como empresarial, a fin de adoptar acciones e implementar estrategias que desde la cultura organizacional brinden los lineamientos necesarios para atender eficientemente las actividades y procesos donde se identifican las fallas.

Si bien los programas de incentivos pueden mejorar la motivación y el empoderamiento del personal en la adopción de herramientas de mejora continua como la filosofía lean, su implementación solo será exitosa si dichas remuneraciones logran incorporarse en la cultura organizacional. Es decir, si las acciones y propuestas presentadas por los individuos van más allá del interés por la remuneración, encaminándose en una mejora global y real del desempeño de la compañía.

Algunas de las empresas participantes que decidieron adoptar la filosofía lean se rigen estrictamente por lo que dictamine el mercado, $y$ otras expresan mayor preocupación por optimizar los procedimientos internos a fin de incrementar la productividad, rentabilidad y calidad. Por ende, la elección de las herramientas a implementar debe ligarse a las necesidades y características de cada proceso productivo o administrativo. No deben desperdiciarse capacidades y recursos en actividades donde estos no son necesarios.

\section{Referencias}

Aguirre, A. M. (2010). Aplicación de metodología seis sigma para mejorar la capacidad de proceso de la variable nivelación vertical en la aplicación de pintura (fondos) de una ensambladora de vehículos. Recuperado de: http://www.bdigital.unal. edu.co/3561/1/anamariaaguirrealvarado.2010.pdf

Alukal, G. y Manos, A. (2006). Lean Kaizen: A Simplified Approach to Process Improvements. (A. Hytinen \& P. O’Mara, Eds.). Milwaukee, USA: William A. Tony. Recuperado de: http://books.google.com/ books?id=aohFNzE0BWkC\&pgis=1 Arrieta, J. G., Botero, V. E. y Romano, M. J. (2011). Benchmarking 
methodologyonlean manufacturing in the apparel industry. In 21st International Conference on Production Research: Innovation in Product and Production, ICPR 2011 - Conference Proceedings. Fraunhofer-Verlag. Recuperado de: http://www.scopus. com/inward/record.url?eid=2-s2.084923485955\&partnerID =tZO tx3y1

Beau Drew, K. A. L. (2004). The Complete Lean Enterprise: Value Stream Mapping for Administrative and Office Processes. Boca Raton, FL: C. Press.

Cardona, J. J. (2013). Modelo Para La Implementación De Técnicas Lean Manufacturing En Empresas Editoriales.

Chang, C.-C., Wu, T.-H. y Wu, C.W. (2013). An efficient approach to determine cell formation, cell layout and intracellular machine sequence in cellular manufacturing systems. Computers \& Industrial Engineering, 66(2), 438-450. http://doi. org/10.1016/j.cie.2013.07.009

Conner, G. (2006). Don't let wasteful tasks slow you down: Lean manufacturing eliminates meaningless activities from the shop floor while boosting productivity and efficiency. Metal
Finishing, 104(3), 13-15. http://doi. org/10.1016/S0026-0576(06)80048-6 Cuatrecasas, L. (2006). Claves de Lean Management. Un enfoque para la alta competitividad en un mundo globalizado. Barcelona, España: Ediciones Gestión. Recuperado de: https://books.google.com.co/ books?id=QRO2lQNXe70C\&print $\mathrm{sec}=$ frontcover\&dq=cuatrecasas $+\mathrm{cl}$ aves+del+lean +management\&hl=e s-419\&sa $=X \& e i=j T R J V c r P N o u u g g$ Tx4IKwBQ\&ved=0CB0QuwUwAA $\# \mathrm{v}=$ onepage\& $\mathrm{q}=$ cuatrecasas claves del lean management $\& f=$ false

Cuatrecasas, L. (2010). TPM en un entorno Lean management estrategia competitiva. Barcelona: Profit.

David, F. R. (2003). Conceptos de administración estratégica. Pearson Educación.

Eckes, G. (2006). El Six Sigma para todos. Editorial Norma. Retrieved from http://books.google.com.co/books/ about/El_Six_Sigma_para_todos. html?id=wcPl_I47fk4C\&pgis=1

Fernández-Ríos, M., y Sánchez, J. C. (1997). Eficacia organizacional: concepto, desarrollo y evaluación. Ediciones Díaz de Santos. 
Gaither, N. (2000). Administración de producción y operaciones. México, D.F.: Thomson.

Garri, M., Konstantopoulos, N. y Bekiaris, M. (2013). Corporate Strategy, Corporate Culture \& Customer Information. Procedia - Social and Behavioral Sciences, 73, 669-677. http:// doi.org/10.1016/j.sbspro.2013.02.104 Guillén, M. del C. M. (2013). Motivación: La gestión empresarial. Ediciones Díaz de Santos.

Hay, E. J. (1989). Justo a tiempo (Just in Time): la técnica japonesa que genera mayor ventaja. Bogotá: Editorial Norma.

Hax, A. C. (2004). Estrategias para el liderazgo competitivo. Ediciones Granica S.A.

Heizer, J. y Render, B. (2007). Dirección de la producción y de operaciones: decisiones estratégicas (Octava Ed.). Madrid: Pearson educación.

Hernández, J. C. y Vizán, A. (2013). Lean manufacturing Conceptos, técnicas e implantación. Madrid: Fundación EOI. Recuperado de: http:// www.eoi.es/savia/documento/

Imai, M. (2012). Gemba Kaizen: A Commonsense Approach to a
Continuous Improvement Strategy, Second Edition (2nd ed.). New York: McGraw Hill.

Jajodia, S., Minis, I., Harhalakis, G. y Proth, J. M. (1992). CLASS. Computerized LAyout Solutions using Simulated annealing. International Journal of Production Research, 30(1), 95-108. Recuperado de: http://www.scopus.com/ inward/record.url? eid=2 - s2.0 0026765726\&partnerID=tZOtx3y1

Kilpatrick, J. (2003). Lean Principles. Manufacturing Extension Partnership, 1-5.

Krajewski, L. J. y Ritzman, L. P. (2000). Administración de operaciones: estrategia y análisis. Pearson Educación.

Maarof, M. G. y Mahmud, F. (2016). A Review of Contributing Factors and Challenges in Implementing Kaizen in Small and Medium Enterprises. Procedia Economics and Finance, 35, 522-531. http://doi.org/10.1016/ S2212-5671(16)00065-4

Madariaga, F. (2013). Lean manufacturing: Exposición adaptada a la fabricación repetitiva de familias de productos mediantre procesos directos.Bubok Publishing S.L. Recupe- 
rado de: https://books.google.com/ books?id=mBgDGYRQzXMC\& pgis $=1$

Maroto, J. C. (2007). Estrategia: de la visión a la acción. ESIC Editorial. Monden, Y. (1996). El just in time hoy en Toyota ( $2^{\mathrm{a}}$ ed.). Bilbao: Ediciones Deusto S.A.

Nash, M. y Poling, S. (2008). Mapping the total value stream: a comprehensive guide for production and transactional processes. CRC Press.

Netland, T. H. (2015). Critical success factors for implementing lean production: the effect of contingencies. International Journal of Production Research, 0(0), 1-16. http:// doi.org/10.1080/00207543.2015.10 96976

Paredes, A. P. (2005). Cultura de la innovación y la gestióntecnológica para el desarrollo de los pueblos. Convenio Andrés Bello.

Pérez Rave, J. (2011). El avión de la muda: herramienta de apoyo a la enseñanza-aprendizaje práctico de la manufactura esbelta. Revista $\mathrm{Fa}$ cultad de Ingeniería, (58), 173-182.

Rajadell, M. y Sánchez, J. L. (2010). Lean Manufacturing. La evidencia de una necesidad. Madrid: Ediciones Díaz de Santos. Recuperado de: http://books.google.com. co/books/about/Lean_Manufacturing_La_evidencia_de_una_n. html?id=lR2xgsdmdUoC\&pgis=1

Rother, M., \& Shook, J. (2003). Learning to See Value Stream Mapping to Create Value and Eliminate Muda. Lean Enterprise Institute Brookline.

Salazar, A. F., Vargas, L. C., Añasco, C. E. y Orejuela, J. P. (2010). Proposta de distribuição em planta bietapa em ambientes de manufatura flexível mediante o processo analítico hierárquico. Revista EIA. Escuela de Ingeniería de Antioquia. Recuperado de: http://dialnet.unirioja.es/servlet/articulo? codigo $=3669$ 801\&info=resumen\&idioma $=$ POR

Sánchez, J. (2007). Propuesta para la implementación del mantenimiento total productivo (TPM). El Cid Editor - Ingeniería.

Santos, Z. G. dos, Vieira, L. y Balbinotti, G. (2015). Lean Manufacturing and Ergonomic Working Conditions in the Automotive Industry. Procedia Manufacturing, 3, 59475954. http://doi.org/10.1016/j. promfg.2015.07.687 
Sullivan, W. G., McDonald, T. N. Wahab, A. N. A., Mukhtar, M. y y Van Aken, E. M. (2002). Equip- Sulaiman, R. (2013). A Concepment replacement decisions and tual Model of Lean Manufacturlean manufacturing. In Robotics ing Dimensions. Procedia Techand Computer-Integrated Manufacturing 18, 255-265. nology, 11, 1292-1298. http://doi. org/10.1016/j.protcy.2013.12.327

Sun, L.-Y., Zhang, Z., Qi, J. y Chen, Z.X. (2012). Empowerment and creativity: A cross-level investigation. The Leadership Quarterly, 23(1), 55-65. http://doi.org/10.1016/j. leaqua.2011.11.005

Tessem, B. (2014). Individual empowerment of agile and non-agile software developers in small teams. Information and Software Technology, 56(8), 873-889. http://doi. org/10.1016/j.infsof.2014.02.005

Villaseñor, A. y Galindo, E. (2007). Manual de Lean Manufacturing. Youssouf, A., Rachid, C. e Ion, V. (2014). Contribution to the Optimization of Strategy of Maintenance by Lean Six Sigma. Physics Procedia, 55, 512-518. http://doi. org/10.1016/j.phpro.2014.08.001

Zavalla, C. A. (2010). Definir, medir, analizar, mejorar y controlar la cadena logística. Énfasis Logística, (5), 66-70. Recuperado de: http:// web.austral.edu.ar/descargas/australis/jul_01_10/medios-definirmedir-analizar-mejorar-y-controlar-la-cadena-logistiica.pdf Guía básica.

Villaseñor, A. y Galindo, E. (2011). Sistema 5 S. Guía de implementación. México, D.F. 